\title{
A METHOD OF SYMMETRIZATION AND APPLICATIONS
}

\author{
BY \\ DOV AHARONOV AND W. E. KIRWAN( $\left.{ }^{1}\right)$
}

\begin{abstract}
In this paper we define a method of symmetrization for plane domains that includes as special cases methods of symmetrization considered by Szegö and by Marcus. We prove that under this method of symmetrization the mapping radius of a fixed point is not decreased. This fact is used to obtain some results concerning covering properties of Bieberbach-Eilenberg functions.
\end{abstract}

1. Introduction. In this paper we define a method of symmetrization for plane domains that generalizes methods of symmetrization considered by Szegö [6] and Marcus [4]. Our main result is that under this method of symmetrization, the mapping radius at a fixed point is not decreased. We will also show that this method of symmetrization preserves certain classes of domains. We conclude with some applications concerning covering properties for the class of BieberbachEilenberg functions.

2. Definitions and remarks. The notation used in defining our method of symmetrization will, as far as possible, follow that used by Marcus [4].

Let $D$ be a domain in the plane. In the sequel there will be no loss of generality to assume that $0 \in D$. If the disk $|z|<\rho$ is contained in $D$ then, following Marcus, we define

$$
L_{\rho}(\phi)=\int_{E} \frac{d r}{r}
$$

where $E=E_{\rho}(\phi)$ is the intersection of $D$ with the set $\{z|| z \mid \geqq \rho, \arg z=\phi\}$,

$$
R(\phi)=\rho \exp L_{\rho}(\phi)
$$

$R(\phi)$ is of course independent of $\rho$.

Let $A=\left\{\alpha_{k}\right\}_{k=1}^{n}$ and $B=\left\{\beta_{k}\right\}_{k=1}^{n}$ be two sequences of real numbers with $\left|\alpha_{k}\right|=1$. We define

$$
R^{(n)}(\phi)=R^{(n)}(\phi, A, B)=\left[\prod_{k=1}^{n} R\left(\alpha_{k} \phi+\beta_{k}\right)\right]^{1 / n}
$$

Received by the editors August 3, 1970 and, in revised form, April 15, 1971.

AMS 1969 subject classifications. Primary 3040; Secondary 3041, 3042, 3052.

Key words and phrases. Conformal mapping, mapping radius, symmetrization, univalent functions, Bieberbach-Eilenberg functions.

(1) The work of this author was supported in part by NSF GP-12547.

Copyright (c) 1972, American Mathematical Society 
DEFINITION 2.1. Let $D$ be a domain in the plane which contains the origin. The $P_{n}[A, B]=P_{n}$ symmetrized set of $D$, denoted $P_{n} D$, is defined by

$$
P_{n} D=\left\{z \mid z=r e^{i \phi}, 0 \leqq r<R^{(n)}(\phi), 0 \leqq \phi<2 \pi\right\} .
$$

RemarKs. (1) It follows immediately from the definition that $P_{n} D$ is a simply connected domain in the plane which is starlike with respect to the origin.

(2) If in Definition 2.1 we take $\alpha_{k}=1(1 \leqq k \leqq n)$ and $\beta_{k}=2 \pi / n$, then $P_{n}$ symmetrization reduces to the method of symmetrization introduced by Marcus [4].

(3) If $D$ is a starlike domain (here and in the sequel starlike means starlike with respect to 0 ) then in (2.2), $R(\phi)$ is just the distance from 0 to the boundary of $D$ along the ray $\arg z=\phi$. Thus if the boundary of $D$ has the polar representation $r=r(\phi)(0 \leqq \phi<2 \pi)$, then $r(\phi)=R(\phi)$. Thus for starlike domains, $P_{n}$ symmetrization generalizes the method of symmetrization considered by Szegö [6].

(4) If we apply Marcus' method of symmetrization with $n=1$ (i.e. radial symmetrization) to $D$, then the symmetrized domain $D^{*}$ is defined by

$$
D^{*}=\left\{z \mid z=r e^{i \phi}, 0 \leqq r<R(\phi), 0 \leqq \phi<2 \pi\right\},
$$

where $R(\phi)$ is defined by (2.2). $D^{*}$ is a starlike domain. If we now apply $P_{n}$ symmetrization to $D^{*}$ we obtain precisely the same domain as if we had originally applied $P_{n}$ symmetrization to $D$. Thus $P_{n}$ symmetrization defined above is a composition of radial symmetrization as defined by Marcus and $P_{n}$ symmetrization for starlike domains. This observation will be important for the proof of our main theorem. Using the special case of the results of Marcus for radial symmetrization, we can reduce the proof of the main theorem to a consideration of $P_{n}$ symmetrization for starlike domains.

(5) In Definition 2.1 the case $n=2, \alpha_{1}=1, \alpha_{2}=-1, \beta_{1}=0$ and $\beta_{2}=0$ will be particularly important for our applications. The symmetrized domain $P_{2} D$ in this case is not only starlike with respect to 0 , but has the additional property that the distances from 0 to the boundary of $P_{2} D$ along the rays $\arg z= \pm \phi(0<\phi<\pi)$ are equal.

3. Main theorem. Let $f(z)$ be analytic in the unit disc, $\mathscr{U}$, and let $D=f(\mathscr{U})$. Without loss of generality we may assume $f(0)=0$. If $f(z)$ is univalent then the mapping radius of $D$ at $0, r(D, 0)=r(D)$, is defined by

$$
r(D)=\left|f^{\prime}(0)\right| \text {. }
$$

If $f(z)$ is not univalent, then $r(D)$ is defined by a limiting process (see [3, p. 79]). Hayman has shown $[3$, p. 80$]$ that

$$
\left|f^{\prime}(0)\right| \leqq r(D)
$$

with equality if and only if $f(z)$ is univalent. A very powerful tool in the theory of conformal mapping is the fact that if $D^{*}$ is obtained from $D$ by Pólya, Steiner or Marcus symmetrization then

$$
r(D) \leqq r\left(D^{*}\right)
$$


(see [3, p. 81] and [4, Theorem 3]). We will now prove the analogous result for the method of symmetrization given in Definition 2.1. The proof uses (3.2) for the special case of Marcus' radial symmetrization and a method due to Szegö.

THeOREM 3.1. Let $f(z)$ be analytic in $\mathscr{U}$ with $f(0)=0$ and let $D=f(\mathscr{U})$. If $P_{n} D$ is the $P_{n}$ symmetrized domain of $D$ and $P_{n} D$ is not the whole plane, then $r(D) \leqq r\left(P_{n} D\right)$.

Proof. We recall Remark 4 of $\S 2 . P_{n} D$ is obtained from $D$ by a composition of Marcus symmetrization with $n=1, S_{1}$, (i.e. radial symmetrization) and $P_{n}$ symmetrization for starlike domains. If $P_{n} D$ is not the plane then $S_{1} D$ is not the plane and hence $r(D) \leqq r\left(S_{1} D\right)$ [4, Theorem 3]. Thus it suffices to show that $P_{n}$ symmetrization for starlike domains does not decrease the mapping radius. To this end, we may suppose that the boundary of $D$ is an analytic Jordan curve. Indeed if not, and $f(z)$ maps $\mathscr{U} 1-1$ onto $D$ with $f(0)=0$, then consider the domain $D_{s}(0<s<1)$ which is the image of $\mathscr{U}$ under $f(s z) . D_{s}$ is starlike with respect to 0 , is bounded by an analytic Jordan curve and

$$
\lim _{s \rightarrow 1} r\left(D_{s}\right)=r(D) \text {. }
$$

Consequently if we have proved the result for domains with an analytic boundary, then $r\left(P_{n} D\right) \geqq r\left(P_{n} D_{s}\right)$ (by the principle of subordination) which together with (3.3) implies $r\left(P_{n} D\right) \geqq r(D)$.

In order to prove our results for starlike domains with analytic boundaries, we will need to make use of some terminology and results concerning condensers in the plane. The reader is referred to [3, Chapter 4] as a convenient reference.

Let $E_{0}$ be the complement of $D$ and $E_{1}$ a small closed disc with center 0 contained in $D$. Using a standard argument [3, p. 83] we will deduce our result if we can show that $P_{n}$ symmetrization decreases the capacity of the condenser $\left(D, E_{0}, E_{1}\right)$. The capacity of a condenser is defined as

$$
I_{D}(\omega)=\iint_{D}\left[\left(\frac{\partial \omega}{\partial x}\right)^{2}+\left(\frac{\partial \omega}{\partial y}\right)^{2}\right] d x d y
$$

where $\omega(z)$ is harmonic in $D-E_{1}$, continuous on $\left(D-E_{1}\right)^{-}$with values 0 on $E_{0}$ and 1 on $E_{1}$. A fundamental fact that our proof requires is that if $u(z)$ is continuous in the extended plane, 0 on $E_{0}, 1$ on $E_{1}$ and is Lipschitz on compact subsets of $D$, then

$$
I_{D}(u) \geqq I_{D}(\omega)
$$

(see for example [3, p. 65]).

Consider the condenser $\left(P_{n} D, P_{n} E_{0}, P_{n} E_{1}\right)$ where $P_{n} E_{0}$ is the complement of $P_{n} D$ and $P_{n} E_{1}=E_{1}$. We will compare the capacity $c^{*}$ of this condenser with the capacity $c$ of $\left(D, E_{0}, E_{1}\right)$. Changing to polar coordinates, we have from (3.4) that

$$
I_{D}(\omega)=\iint_{D}\left[\left(\frac{\partial \omega}{\partial r}\right)^{2}+\frac{1}{r^{2}}\left(\frac{\partial \omega}{\partial \phi}\right)^{2}\right] r d r d \phi
$$


Let $L_{\lambda}$ denote the $\lambda$ level curve of $\omega(z)$, i.e., $L_{\lambda}=\{z \mid \omega(z)=\lambda, 0 \leqq \lambda \leqq 1\}$. Since $D$ is a starlike domain, $L_{\lambda}$ is starlike with respect to 0 [6, p. 115]. Let $r=r(\lambda, \phi)$ be the polar equation of $L_{\lambda}$. On $L_{\lambda}$ we have

$$
\omega(r(\lambda, \phi) ; \phi) \equiv \lambda
$$

and hence $\omega_{r} r_{\lambda}=1$ and $\omega_{r} r_{\phi}+\omega_{\phi}=0$. Substituting in (3.6), we obtain

$$
\begin{aligned}
I_{D}(\omega) & =\int_{0}^{1} \int_{-\pi}^{\pi}\left[r_{\lambda}^{-2}+r^{-2}\left(r_{\phi} / r_{\lambda}\right)^{2}\right] r r_{\lambda} d \phi d \lambda \\
& =\int_{0}^{1} \int_{-\pi}^{\pi} \frac{1+\left(r_{\phi} / r\right)^{2}}{r_{\lambda} / r} d \phi d \lambda .
\end{aligned}
$$

For future reference we set

$$
I_{\lambda}=\int_{-\pi}^{\pi} \frac{1+\left(r_{\phi} / r\right)^{2}}{r_{\lambda} / r} d \phi
$$

The curves $L_{\lambda}$ correspond to the curves $L_{\lambda}^{*}$ in the symmetrized condenser $\left(P_{n} D, P_{n} E_{0}, P_{n} E_{1}\right)$ where $L_{\lambda}^{*}$ is the curve with polar equation

$$
\rho=\rho(\lambda, \phi)=\left[\prod_{k=1}^{n} r\left(\lambda, \alpha_{k} \phi+\beta_{k}\right)\right]^{1 / n} .
$$

Since the $L_{\lambda}$ are analytic and starlike, it is clear that the $L_{\lambda}^{*}$ are analytic and mutually disjoint. We define $\omega^{*}(z)$ in the extended plane by taking $\omega^{*}(z)=0$ on $P_{n} E_{0}, \omega^{*}(z)=1$ on $P_{n} E_{1}$ and

$$
\omega^{*}(\rho(\lambda, \phi) ; \phi)=\lambda
$$

for $z=\rho(\lambda, \phi) e^{i \phi}$ in $P_{n} D-P_{n} E_{1}$. It follows that $\omega^{*}(z)$ is continuous in the extended plane, 0 on $P_{n} E_{0}, 1$ on $P_{n} E_{1}$ and Lipschitz on compact subsets of $P_{n} D-P_{n} E_{1}$ (in fact (3.7), (3.9) and (3.10) show that $\omega^{*}$ is analytic in $\left.P_{n} D-P_{n} E_{1}\right)$. Consequently from (3.5) we have that $I_{P_{n} D}\left(\omega^{*}\right)$ is larger than the capacity of $\left(P_{n} D, P_{n} E_{0}, P_{n} E_{1}\right)$, and so it remains to show that

$$
I_{P_{n} D}\left(\omega^{*}\right) \leqq I_{D}(\omega)
$$

To show this inequality we use a modification of an argument due to Szegö [6]. Repeating the argument used to obtain (3.8), we have

$$
I_{P_{n} D}\left(\omega^{*}\right)=\int_{0}^{1} \int_{-\pi}^{\pi} \frac{1+\left(\rho_{\phi} / \rho\right)^{2}}{\rho_{\lambda} / \rho} d \lambda d \phi
$$

where $\rho$ is given by (3.9).

Now, let

$$
\begin{aligned}
I_{\lambda}^{*} & =\int_{-\pi}^{\pi} \frac{1+\left(\rho_{\phi} / \rho\right)^{2}}{\rho_{\lambda} / \rho} d \phi \\
& =\int_{-\pi}^{\pi} \frac{1+\left[(1 / n) \sum_{k=1}^{n} \alpha_{k} r_{\phi}\left(\lambda, \alpha_{k} \phi+\beta_{k}\right) / r\left(\lambda, \alpha_{k} \phi+\beta_{k}\right)\right]^{2}}{(1 / n) \sum_{k=1}^{n} r_{\lambda}\left(\lambda, \alpha_{k} \phi+\beta_{k}\right) / r\left(\lambda, \alpha_{k} \phi+\beta_{k}\right)} d \phi .
\end{aligned}
$$


In [6] Szegö proved the inequality

$$
\frac{1}{n} \sum_{k=1}^{n} \frac{1+x_{k}^{2}}{\left|y_{k}\right|} \geqq \frac{1+\left((1 / n) \sum_{k=1}^{n}\left|x_{k}\right|\right)^{2}}{(1 / n) \sum_{k=1}^{n}\left|y_{k}\right|}
$$

We set

$$
x_{k}=\frac{\alpha_{k} r_{\phi}\left(\lambda, \alpha_{k} \phi+\beta_{k}\right)}{r\left(\lambda, \alpha_{k} \phi+\beta_{k}\right)}, \quad y_{k}=\frac{r_{\lambda}\left(\lambda, \alpha_{k} \phi+\beta_{k}\right)}{r\left(\lambda, \alpha_{k} \phi+\beta_{k}\right)} .
$$

Since $L_{\lambda}$ is starlike, $r_{\lambda}>0$ and hence it follows from (3.13) and (3.14) (recall that $\left|\alpha_{k}\right|=1$ ) that

$$
I_{\lambda}^{*} \leqq \frac{1}{n} \sum_{k=1}^{n} \int_{-\pi}^{\pi} \frac{1+\left[r_{\phi}\left(\lambda, \alpha_{k} \phi+\beta_{k}\right) / r\left(\lambda, \alpha_{k} \phi+\beta_{k}\right)\right]^{2}}{\left[r_{\lambda}\left(\lambda, \alpha_{k} \phi+\beta_{k}\right) / r\left(\lambda, \alpha_{k} \phi+\beta_{k}\right)\right]} d \phi .
$$

Since $r(\lambda, \phi)$ is periodic with period $2 \pi$, each integral on the right side of the above inequality equals

$$
I_{\lambda}=\int_{-\pi}^{\pi} \frac{1+\left[r_{\phi}(\lambda, \phi) / r(\lambda, \phi)\right]^{2}}{r_{\lambda}(\lambda, \phi) / r(\lambda, \phi)} d \phi .
$$

Thus $I_{\lambda}^{*} \leqq I_{\lambda}$. Integrating this inequality from 0 to 1 and recalling (3.8) and (3.12), we see that (3.11) is established and the proof is complete.

REMARK. With minor modifications in the above argument, we can prove that, more generally, if $\left(D, E_{0}, E_{1}\right)$ is an admissible condenser, $\left(P_{n} D, P_{n} E_{0}, P_{n} E_{1}\right)$ is the $P_{n}$ symmetrized condenser, and if these condensers have respective capacities $c$ and $c_{n}$, then $c_{n} \leqq c$.

4. Applications for Bieberbach-Eilenberg functions. Let $f(z)=\sum_{n=1}^{\infty} a_{n} z^{n}$ be analytic in $\mathscr{U}=\{|z|<1\} . f(z)$ is a Bieberbach-Eilenberg function if $f(z) \cdot f(w) \neq 1$ for any $|z|<1$ and $|w|<1$. $\mathscr{B}$ will denote the class of all such functions. We will show that with a suitable choice of the sequences $\left\{\alpha_{k}\right\}$ and $\left\{\beta_{k}\right\}, P_{n}$ symmetrization preserves the class $\mathscr{B}$. First we prove the following lemma the elegant proof of which was supplied by the referee who greatly simplified the authors' original proof.

Lemma. Let $f(z) \in \mathscr{B}$ and $D=f(\mathscr{U})$. Then

$$
R(\phi) \cdot R(-\phi) \leqq 1 \quad(0 \leqq \phi<2 \pi)
$$

where $R(\phi)$ is defined by (2.2).

Proof. Suppose that $D$ contains the disk $|w|<\rho$, so that $D$ lies in $|w|<1 / \rho$. Let $E_{1}, E_{2}$ denote the sets of real $t$, such that $\rho<t<1 / \rho$ and $t e^{i \phi} \in D, t e^{-i \phi} \in D$ respectively. Let $E_{1}^{*}$ consist of all $t$ for which $t^{-1} \in E_{1}$. Then by the hypothesis $E_{1}^{*}$ and $E_{2}$ are disjoint. Thus

$$
\int_{E_{1}} \frac{d t}{t}+\int_{E_{2}} \frac{d t}{t}=\int_{E_{1}^{*}} \frac{d t}{t}+\int_{E_{2}} \frac{d t}{t} \leqq \int_{\rho}^{1 / \rho} \frac{d t}{t}=2 \log \frac{1}{\rho}
$$


Hence

$$
\log R(\phi)+\log R(-\phi)=\log \rho+\int_{E_{1}} \frac{d t}{t}+\log \rho+\int_{E_{2}} \frac{d t}{t} \leqq 0
$$

and the proof is complete.

THEOREM 4.1. Let $f(z) \in \mathscr{B}$ and $D=f(\mathscr{U})$. Let $n$ be a positive integer and $A=$ $\left\{\alpha_{k}\right\}_{k=1}^{2 n}, B=\left\{\beta_{k}\right\}_{k=1}^{2 n}$ be two sequences of real numbers with $\left|\alpha_{k}\right|=1, \alpha_{n+k}=-\alpha_{k}$ and $\beta_{n+k}=-\beta_{k}(1 \leqq k \leqq n)$. If $P_{2 n}=P_{2 n}(A, B)$ and $g(z)=a_{1} z+a_{2} z^{2}+\cdots\left(a_{1}>0\right)$ is the conformal map of $\mathscr{U}$ onto $P_{2 n} D$, then

(i) $\left|g^{\prime}(0)\right| \geqq\left|f^{\prime}(0)\right|$,

(ii) $|g(z)|<1$ for $z \in \mathscr{U}$ (in particular $g(z) \in \mathscr{B})$,

(iii) $g(\mathscr{U})$ is starlike.

\section{Proof. From Definition 2.1}

where

$$
P_{2 n} D=\left\{w \mid w=r e^{i \phi}, 0 \leqq r<R^{(2 n)}(\phi)\right\}
$$

$$
\begin{aligned}
R^{(2 n)}(\phi) & =\left[\prod_{k=1}^{n} R\left(\alpha_{k} \phi+\beta_{k}\right) \prod_{k=1}^{n} R\left(-\alpha_{k} \phi-\beta_{k}\right)\right]^{1 / 2 n} \\
& =\left[\prod_{k=1}^{n} R\left(\alpha_{k} \phi+\beta_{k}\right) R\left(-\alpha_{k} \phi-\beta_{k}\right)\right]^{1 / 2 n} .
\end{aligned}
$$

By the lemma $R^{(2 n)}(\phi) \leqq 1$. This proves (ii). (iii) is immediate and (i) follows from (3.1) and Theorem 3.1.

REMARK. Another consequence of the lemma is that Marcus' method of radial symmetrization, $S_{1}$, (see Remark 4 of $\S 2$ ) preserves Bieberbach-Eilenberg domains. Indeed if $D=f(\mathscr{U})$ where $f \in \mathscr{B}$ then

$$
S_{1} D=\left\{w \mid w=r e^{i \phi}, 0 \leqq r<R(\phi)\right\} .
$$

$S_{1} D$ is a starlike domain. Using this fact, it is easy to see that since $R(\phi) \cdot R(-\phi) \leqq 1$, the function $g(z)$ that maps $\mathscr{U} 1-1$ onto $S_{1} D$ and satisfies $g(0)=0$ belongs to $\mathscr{B}$.

As an application of Theorem 4.1, we consider a question that was raised by Fekete for the class $\mathscr{S}$ (i.e. the class of analytic and univalent functions in $\mathscr{U}$ with the usual normalization) [2] and solved for this class by Marcus [4]. For the class $\mathscr{B}$ the problem takes the following form. Let $\mathscr{B}(\rho)(0<\rho \leqq 1)$ denote those functions $f(z) \in \mathscr{B}$ with $r(f(\mathscr{U}))=\rho .(r(f(\mathscr{U}))$ denotes the mapping radius of $f(\mathscr{U})$ with respect to 0 .) Given $n$ rays issuing from the origin at equal angles $2 \pi / n$, let $L=L(f)$ denote the linear measure of the intersection of these rays with $f(\mathscr{U})$. What is the minimum value of $L$ over the class $\mathscr{B}(\rho)$ ? In this direction we have the following result.

THEOREM 4.2. Let $f(z) \in \mathscr{B}(\rho)$ and let $l_{k}=l_{k}(f)$ denote the linear measure of the intersection of $f(\mathscr{U})$ with the ray $\arg w=2 k \pi / n(0 \leqq k \leqq n-1)$. Then

$$
L(f)=\sum_{k=1}^{n} l_{k} \geqq n\left[\prod_{k=1}^{n} l_{k}\right]^{1 / n} \geqq n\left[2-\rho^{n}-2\left(1-\rho^{n}\right)^{1 / 2}\right]^{1 / n} / \rho
$$

and there is a function in $\mathscr{B}(\rho)$ for which equality holds throughout (4.1). 
Proof. It is easy to show (see for example [5, p. 224]) that the function $h(z)$ defined by

$$
h(z) /(1+h(z))^{2}=\rho^{n} z /(1+z)^{2} \quad(0<\rho<1)
$$

maps $\mathscr{U}$ conformally onto $\{|w|<1\}$ minus the interval $[\lambda(\rho), 1)$ where

$$
\lambda(\rho)=\left(2-\rho^{n}-2\left(1-\rho^{n}\right)^{1 / 2}\right) / \rho^{n} .
$$

Clearly $h(0)=0$ and $h^{\prime}(0)=\rho^{n}$ so that $h(z) \in \mathscr{B}\left(\rho^{n}\right)$. We define

$$
\diamond(z)=\left[h\left(z^{n}\right)\right]^{1 / n} .
$$

$\diamond(z) \in \mathscr{B}(\rho)$ and maps $\mathscr{U}$ conformally onto $\{|w|<1\}$ minus the $n$ segments

$$
\left[[\lambda(\rho)]^{1 / n} e^{2 k \pi i / n}, e^{2 k \pi i / n}\right) \quad(0 \leqq k \leqq n-1) .
$$

For the function $s(z)$, equality holds throughout (4.1). It remains to show that if $f \in \mathscr{B}(\rho)$ then

$$
L(f) \geqq L(s) .
$$

Let $f \in \mathscr{B}(\rho)$ and $D=f(\mathscr{U})$. We will prove (4.2) by successively applying three special cases of our method of symmetrization. At each stage we will show that the corresponding $L$ has been decreased. First we apply Marcus' method of radial symmetrization to $D$, obtaining $S_{1} D$, where

$$
S_{1} D=\left\{w \mid w=r e^{i \phi}, 0 \leqq r<R(\phi), 0 \leqq \phi<2 \pi\right\} .
$$

By the result of Marcus cited in (3.2), $r(D) \leqq r\left(S_{1} D\right)$. Let $g_{1}(z)$ be the conformal map of $\mathscr{U}$ onto $S_{1} D$ with $g_{1}(0)=0$ and $g_{1}^{\prime}(0)>0$. (In the sequel we will refer to the conformal map, $\tau(z)$, of $\mathscr{U}$ onto a domain $H$ with the normalization $\tau(0)=0, \tau^{\prime}(0)>0$ as the associated mapping function of $H$.) $g_{1}(z)$ satisfies $\rho_{1}=g_{1}^{\prime}(0)=r\left(S_{1} D\right) \geqq \rho$. By the remark following Theorem 4.1, $g_{1}(z) \in \mathscr{B}$. For each $k, 0 \leqq k \leqq n-1, l_{k}\left(g_{1}\right)=$ $R(2 k \pi / n)$. Marcus has shown [4, p. 625] that for an arbitrary domain $D, R(\phi) \leqq l(\phi)$, where $l(\phi)$ denotes the linear measure of $D \cap\{\arg w=\phi\}$. Hence, $l_{k}\left(g_{1}\right) \leqq l_{k}(f)$ and $L\left(g_{1}\right) \leqq L(f)$. Define

$$
f_{1}(z)=\left(\rho / \rho_{1}\right) g_{1}(z)
$$

Since $g_{1}(z)$ is starlike and $\rho / \rho_{1} \leqq 1, f_{1}(z) \in \mathscr{B}$. In fact, $f_{1}(z) \in \mathscr{B}(\rho)$ since $f^{\prime}(0)=\rho$. Also, $\rho / \rho_{1} \leqq 1$ implies

$$
L\left(f_{1}\right) \leqq L\left(g_{1}\right) \leqq L(f) .
$$

Let $f_{1}(\mathscr{U})=D_{1}$. We now apply the symmetrization of Theorem 4.1 to $D_{1}$ with $n=1, \alpha_{1}=1, \alpha_{2}=-1, \beta_{1}=0=\beta_{2}$.

$$
P_{2} D_{1}=\left\{w \mid w=r e^{i \phi}, 0 \leqq r<R^{(2)}(\phi), 0 \leqq \phi<2 \pi\right\}
$$

where

$$
R^{(2)}(\phi)=(R(\phi) R(-\phi))^{1 / 2}
$$


By Theorem 3.1, $r\left(P_{2} D_{1}\right) \geqq r\left(D_{1}\right)$. Let $g_{2}(z)$ be the associated mapping function of $P_{2} D_{1}$. From Theorem 4.1 we have that $\left|g_{2}(z)\right|<1, g_{2}(z) \in \mathscr{B}$ and $\rho_{2}=g_{2}^{\prime}(0)=r\left(P_{2} D_{1}\right)$ $\geqq r\left(D_{1}\right)=\rho$. From (4.4) it follows that

$$
l_{k}\left(g_{2}\right)=l_{n-k}\left(g_{2}\right)=\left(l_{k}\left(f_{1}\right) l_{n-k}\left(f_{1}\right)\right)^{1 / 2} \quad(0 \leqq k \leqq n-1)
$$

and hence by the arithmetic-geometric mean inequality

$$
L\left(g_{2}\right)=\sum_{k=0}^{n-1} l_{k}\left(g_{2}\right) \leqq \sum_{k=0}^{n-1} l_{k}\left(f_{1}\right)=L\left(f_{1}\right)
$$

Define

$$
f_{2}(z)=\left(\rho / \rho_{2}\right) g_{2}(z)
$$

Repeating the argument given above for $f_{1}(z)$, we see that $f_{2}(z) \in \mathscr{B}(\rho)$ and

$$
L\left(f_{2}\right) \leqq L\left(f_{1}\right) .
$$

Let $f_{2}(\mathscr{U})=D_{2}$. We now apply Szegö's method of $n$-fold symmetrization to $D_{2}$ (see Remark 3 of $\S 2$ ). Let $D_{2}^{*}$ be the resulting domain and $g_{3}(z)$ the associated mapping function. Since $D_{2}$ is contained in $\{|w|<1\}, D_{2}^{*}$ is contained in $\{|w|<1\}$ and therefore $g_{3} \in \mathscr{B}$. By the theorem of Szegö [6] (which is included in Theorem 3.1)

$$
\rho_{3}=g_{3}^{\prime}(0)=r\left(D_{2}^{*}\right) \geqq r\left(D_{2}\right)=\rho .
$$

$D_{2}^{*}$ is $n$-fold symmetric and hence $l_{k}\left(g_{3}\right)=l_{j}\left(g_{3}\right)(0 \leqq k, j \leqq n-1)$. Further,

$$
l_{k}\left(g_{3}\right)=\left(l_{1}\left(f_{2}\right) \cdots l_{n}\left(f_{2}\right)\right)^{1 / n} \quad(0 \leqq k \leqq n-1) .
$$

By the arithmetic-geometric mean inequality we have

$$
L\left(g_{3}\right)=\sum_{k=0}^{n-1} l_{k}\left(g_{3}\right) \leqq \sum_{k=0}^{n-1} l_{k}\left(f_{2}\right)=L\left(f_{2}\right) .
$$

If we set $f_{3}(z)=\left(\rho / \rho_{3}\right) g_{3}(z)$ then

$$
L\left(f_{3}\right) \leqq L\left(f_{2}\right) .
$$

Let $D_{3}=f_{3}(\mathscr{U})$ and let $D_{4}$ be $\{|w|<1\}$ minus the $n$ segments $\left[l\left(f_{3}\right) e^{2 k \pi i / n}, e^{2 k \pi i / n}\right)$ where $l\left(f_{3}\right)=l_{k}\left(f_{3}\right)(0 \leqq k \leqq n-1)$. Let $g_{4}(z)$ be the associated mapping function of $D_{4} . g_{4} \in \mathscr{B}$ and $g_{4}(\mathscr{U})=D_{4} \supset D_{3}$, so by the principle of subordination,

$$
\rho_{4}=g_{4}^{\prime}(0)=r\left(D_{4}\right) \geqq r\left(D_{3}\right)=\rho .
$$

Define

$$
f_{4}(z)=\left(\rho / \rho_{4}\right) g_{4}(z)
$$

$f_{4}(z) \in \mathscr{B}(\rho)$ and again,

$$
L\left(f_{4}\right) \leqq L\left(g_{4}\right)=L\left(f_{3}\right)
$$


From the definition of $g_{4}(\mathscr{U})$ it follows that $f_{4}(z)$ maps the unit disk onto the disk $\left\{|w|<\rho / \rho_{4} \leqq 1\right\}$ slit along the $n$ segments $\left[\left(\rho / \rho_{4}\right) l\left(f_{3}\right) e^{2 k \pi i / n},\left(\rho / \rho_{4}\right) e^{2 k \pi i / n}\right)(0 \leqq k \leqq n-1)$. If $L(\jmath)>L\left(f_{4}\right)$ then it follows that $f_{4}(\mathscr{U})$ is properly contained in $\varsigma(\mathscr{U})$. But this is impossible since $r\left(f_{4}(\mathscr{U})\right)=\rho=r(\mathcal{U}(\mathscr{U}))$. Thus $L(\jmath) \leqq L\left(f_{4}\right)$ and combining (4.3), (4.5), (4.6) and (4.7) we see that (4.2) is established. Since $f \in \mathscr{B}(\rho)$ was arbitrary, the proof is complete.

In conclusion the authors would like to express their appreciation to the referee not only for providing the shorter proof of the lemma in $\$ 4$, but also for pointing out an error in the original draft of the paper.

\section{BIBLIOGRAPHY}

1. E. F. Beckenbach and R. Bellman, Inequalities, Ergebnisse der Mathematik und ihrer Grenzgebiete, Heft 30, Springer-Verlag, Berlin, 1961. MR 28 \#1266.

2. M. Fekete, Uber die Verteilung der Wurzeln bei gewissen algebraischen Gleichungen mit ganzzahligen Koeffizienten, Math. Z. 17 (1923), 228-249.

3. W. K. Hayman, Multivalent functions, Cambridge Tracts in Math. and Math. Phys., no. 48, Cambridge Univ. Press, Cambridge, 1958. MR 21 \#7302.

4. M. Marcus, Transformations of domains in the plane and applications in the theory of functions, Pacific J. Math. 14 (1964), 613-626. MR 29 \#2382.

5. Z. Nehari, Conformal mapping, McGraw-Hill, New York, 1952. MR 13, 640.

6. G. Szegö, On a certain kind of symmetrization and its applications, Ann. Mat. Pura Appl. (4) 40 (1955), 113-119. MR 17, 1074.

Technion, Israel Institute of Technology, Haifa, Israel

University of Maryland, College Park, Maryland 20742 